\title{
« Fab labs », « makerspaces » : entre innovation et émancipation?
}

\section{Fab labs and makerspaces: Between innovation and liberation? «Fab labs», «makerspaces»: ¿entre innovación y emancipación?}

\section{Yannick Rumpala}

Numéro 334, octobre 2014

URI : https://id.erudit.org/iderudit/1027278ar

DOI : https://doi.org/10.7202/1027278ar

Aller au sommaire du numéro

Éditeur(s)

Association Recma

ISSN

1626-1682 (imprimé)

2261-2599 (numérique)

Découvrir la revue

Citer cet article

Rumpala, Y. (2014). « Fab labs ", « makerspaces » : entre innovation et émancipation ? Revue internationale de l'économie sociale, (334), 85-97. https://doi.org/10.7202/1027278ar
Résumé de l'article

Dans le sillage d'une image médiatique de communautés de bricolage high-tech, « fab labs » et " makerspaces » sont fréquemment regardés par le prisme de l'innovation. Cet article propose de montrer qu'une telle vision, plutôt technico-économique, est insuffisante et qu'il est possible de les aborder avec un autre regard, plus attentif à leurs potentialités sociopolitiques. Ces lieux ouverts, qui permettent d'accéder à des capacités de fabrication à partir d'équipements sophistiqués, souvent numériques, paraissent en effet pouvoir incarner des courants de réflexion cherchant des voies d'émancipation avec les développements technologiques. "Fab labs " et " makerspaces " méritent donc d'être analysés, notamment sous l'angle des capacités qui semblent redistribuées, des formes de remise en cause de l'ordre industriel qu'ils tendent à porter et des dynamiques qui peuvent être favorables au développement de cette nouvelle forme d'ateliers. 


\title{
« FAB LABS », « MAKERSPACES »: ENTRE INNOVATION ET ÉMANCIPATION?
}

\author{
par Yannick Rumpala*
}

\begin{abstract}
* Université de Nice, faculté de droit et de science politique, équipe de recherche sur les mutations de l'Europe et de ses sociétés (Ermes). Mél.: rumpala@unice.fr.
\end{abstract}

Dans le sillage d'une image médiatique de communautés de bricolage high-tech, «fab labs » et «makerspaces» sont fréquemment regardés parle prisme de l'innovation. Cet article propose de montrer qu'une telle vision, plutôt technico-économique, est insuffisante et qu'il est possible de les aborder avec un autre regard, plus attentifà leurs potentialités sociopolitiques. Ces lieux ouverts, qui permettent d'accéder à des capacités de fabrication à partir d'équipements sophistiqués, souvent numériques, paraissent en effet pouvoir incarner des courants de réflexion cherchant desvoies d'émancipation avec les développements technologiques. «Fablabs » et « makerspaces» méritent donc d'être analysés, notamment sous l'angle des capacités qui semblent redistribuées, des formes de remise en cause de l'ordre industriel qu'ils tendent à porter et des dynamiques qui peuvent être favorables au développement de cette nouvelle forme d'ateliers.

\section{Fab labs and makerspaces: Between innovation and liberation?}

Presented in the media as high-tech DIY communities, fab labs and makerspaces are often seen through the prism of innovation. This article aims to show that this rather techno-economic view has limitations. They can also be examined from another angle, which is more concerned with their socio-political potential. These open spaces, which provide access to production capacities using sophisticated and often digital equipment, appear to embody schools of thought looking for ways of liberation through technological developments. Fab labs and makerspaces therefore merit to be examined, particularly in how they can redistribute capabilities, challenge the industrial order, and foster the development of this new form of workshop.

« Fab labs », « makerspaces » : ¿ entre innovación y emancipación?

Siguiendo la imagen mediática de comunidades de manipulación high-tech, « fab labs » y « makerspaces » son vistos frecuentemente a través del prisma de la innovación. En el presente artículo se muestra que este tipo de visión, bastante técnica-económica, es insuficiente y que es posible considerar los « fab labs » $\mathrm{y}$ « makerspaces » desde una nueva perspectiva que seria más sensible hacia sus potencialidades socio-políticas. En la medida en que estos espacios abiertos permiten acceder a capacidades de fabricación a partir de equipos sofisticados a menudo numéricos, ellos parecen poder encarnar la búsqueda filosófica de la emancipación con el desarrollo tecnológico. Fablabs y makerspaces merecen pués ser analizados, en particular desde la óptica de las capacidades que parecen ser redistribuidas, de un cierto cuestionamiento del orden industrial que tienden a promover, y de las dinámicas que pueden favorecer al desarrollo de este nuevo tipo de talleres. 
Rattrapés par les enthousiasmes technophiles et l'idéologie entrepreneuriale, les fab labs ("fabrication laboratories») pourraient facilement passer pour un nouvel avatar de l'économie de l'innovation ${ }^{(1)}$. Une espèce de variante des start-up ou la manifestation d'un esprit similaire. Ces lieux de fabrication et surtout d'expérimentation, généralement à base de technologies numériques, sont alors presque assimilés,

(1) Voir par exemple « Les "fab labs", l'avenirdel'innovation", L'Entreprise, 18 octobre 2013. voire réduits, à des incubateurs d'entreprises orientés vers les technologies innovantes. C'est une possibilité, mais parmi d'autres, notamment si on la replace dans une tendance au développement d'une multiplicité d'espaces plus ou moins communautaires visant à partager l'accès à des équipements sophistiqués à vocation productive comme les makerspaces, cet autre type de lieu permettant de se réunir et de collaborer pour créer et fabriquer en commun.

Initialement, les fab labs sont des ateliers orientés vers les nouvelles technologies conçus pour être accessibles à des non-professionnels: ils mettent à disposition des outils avancés, en général plus facilement disponibles dans le monde industriel, pour que leurs utilisateurs puissent fabriquer leurs propres objets. L'idée, inspirée du travail du professeur Neil Gershenfeld à la fin des années 90 au Massachusetts Institute of Technology (MIT; Gershenfeld, 2007) et portée par le laboratoire de ce dernier (le Center for Bits and Atoms), a été reprise dans de nombreux pays, et pas seulement ceux qui sont habituellement considérés comme étant les plus avancés techniquement, puisque des fab labs réputés sont aussi présents au Ghana et en Inde, par exemple ${ }^{(2)}$.

Le mouvement ainsi lancé a eu tendance à être absorbé par tout un discours emphatique, allant jusqu'à annoncer une "nouvelle révolution industrielle »(Anderson, 2012). Mais fab labs et makerspaces (les dénominations recouvrant en fait souvent des démarcations poreuses $\left.{ }^{(3)}\right)$ semblent révéler d'autres potentialités et peuvent aussi représenter une nouvelle forme, actualisée, d'ateliers communautaires ou d'ateliers de quartier. Un penseur critique comme André Gorz, dans ses dernières années, a défendu ce genre d'espoir, dans une forme de réactualisation et de promotion de "l'autoproduction communale coopérative » (Gorz, 2007, p. 105-106; Gorz, 2008, p. 121). La formulation était encore dans le registre de l'hypothèse. Avec le développement notable de ces lieux, celle-ci peutelle trouver davantage de substance? Plus précisément, fab labs et makerspaces peuvent-ils constituer un mode de production (si on les analyse par leurs débouchés) ou un système productif différent (si on les analyse par leur tissu organisationnel et leur inscription spatiale)? Dans quelle mesure? Même s'ils paraissent se situer à l'écart de l'ordre industriel, peuvent-ils l'affecter, le perturber?

(2) « Fab Wiki », le wiki de la « communauté » desfablabs, permet notamment de suivre l'augmentation du nombre d'implantations (du moins celles qui sont enregistrées) à travers le monde (http://wiki. fablab.is/wiki/Portal:Labs). En France, soixante-dix initiatives étaient recensées début août 2014, dont vingt-cinq " en développement » et cinq " en projet".

(3) On ne traitera pas ici des techshops, qui sont une version proche, mais où, dans une logique commerciale, les usagers doivent payer pour l'utilisation des machines, souvent plus professionnelles, et les éventuelles formations. Les potentialités des fab labs (si l'on prolonge une approche en termes de "potentialisme technologique ; Rumpala, 2013a) semblent tenir à l'assemblage qu'ils réalisent: des machines qui se rapprochent des standards professionnels, des modes de fonctionnement basés sur l'ouverture, des cadres relationnels propices aux échanges et à un apprentissage en commun. Comparés à l'ordre industriel, ces lieux laissent entrevoir des possibilités 
de reconfigurations multiples et interreliées, en l'occurrence dans le rapport aux équipements et aux systèmes techniques, au travail et aux fonctionnements organisationnels, à l'espace et aux territoires, aux objets et aux matières, ainsi qu'aux circuits économiques.

Pour mieux appréhender ces potentialités (dépendant donc des types d'acteurs qui s'agrègent aux projets, mais aussi des

(4) Cet article s'appuie sur une recherche en cours sur les processus d'expérimentation d'alternatives sociales àl'écart du marché et de l'Etat, les types d'activités originales qu'elles déploient et les formes d'affinités qu'elles peuvent trouver entre elles malgré leur diversité. intérêts et des valeurs qu'ils vont introduire et pousser, des utilisations et des pratiques qu'ils vont promouvoir), la suite de l'article reviendra d'abord sur les caractéristiques de ces lieux et les promesses qu'ils paraissent ainsi porter ${ }^{(4)}$. On montrera, ensuite, en quoi ces espaces participent d'une redistribution de capacités, pas seulement techniques. Si un système productif paraît presque prendre forme à distance de l'ordre industriel, il s'agira, enfin, d'en évaluer les forces et les implications, y compris politiques.

\section{Une nouvelle forme d'atelier en version high-tech?}

Les espaces mis en service sont au croisement de logiques multiples et de différentes rationalités. L'attraction apparente vers les nouvelles technologies, particulièrement celles qui sont liées à la fabrication, peut recouvrir des motivations diverses, qui ne sont justement pas réductibles à des enjeux technologiques.

\section{Expérimentations émergentes}

Les fab labs s'apparentent à des ateliers, mais se distinguent par les outils qu'ils utilisent (notamment des machines à commande numérique) et par les qualités, voire les valeurs, mises en avant: à la différence du modèle de l'établissement industriel, ils sont conçus pour être ouverts, partagés, collaboratifs. Ils visent à permettre de concevoir, de prototyper, de fabriquer et de tester les objets les plus divers ("presque tout», selon les promesses de Neil Gershenfeld, 2012), avec un équipement de pointe mais accessible à un large public, plus large en tout cas que celui des professionnels déjà capables d'utiliser ce type d'équipement. Des machines potentiellement coûteuses (découpe laser, fraiseuse numérique, imprimante 3D, etc.), en plus du petit outillage complémentaire et de l'équipement informatique pour la conception assistée par ordinateur (CAO), sont mises à disposition pour un usage partagé (dans certains cas avec un « fabmanager » qui assume la responsabilité de l'animation et de la coordination du lieu).

En principe, le label fab lab à proprement parler suppose l'acceptation d'une (courte) charte, qui garde l'empreinte du projet d'origine

(5) Traduction en français consultée le 12 juin 2014 sur http://wiki.fablab.is/wiki/ Fab_Charter/fr. Pour la version originale (les traductions pouvant varier), voir « The Fab Charter », sur http://fab. cba.mit.edu/about/charter. élaboré au MIT. La définition que donne la charte du MIT trace en effet presque déjà un projet collectif: "Les fab labs sont un réseau mondial de labs locaux qui doppent l'inventivité en donnant accès à des outils de fabrication numérique. » En ajoutant aussi : "Les fab labs partagent le catalogue évolutif d'un noyau de capacités pour fabriquer (presque) n'importe quel objet, permettant aux personnes et aux projets d'être partagés » ${ }^{(5)}$. La dénomination "fab lab» a 
cependant largement été reprise en dehors de ce cadre et sans forcément faire référence à la filiation originelle. D’ailleurs, en fonction de la qualité des équipements proposés et donc de leur coût (de quelques milliers de dollars ou d'euros à quelques centaines de milliers pour les plus gros fab labs), les configurations diffèrent et peuvent rendre les projets plus ou moins facilement réalisables. Les modèles d'organisation peuvent également être variés, selon leur orientation vers le secteur de l'enseignement, vers les entreprises ou vers le grand public (même, potentiellement, avec une orientation militante). Ils laissent à chaque fois entrevoir des généalogies différenciées, mais aussi des intérêts et des valeurs encore hétérogènes. Fabien Eychenne (2012) avait tenté une typologie, en distinguant trois types de fab labs. Ceux de type "éducationnel » sont reliés à des établissements d'enseignement, comme le Faclab de l'université de Cergy-Pontoise, qui a même élaboré trois diplômes universitaires (DU) dans le prolongement du projet initial. Ceux de type "privé-business » traduisent plutôt les intérêts des entreprises qui en sont à l'origine, généralement dans une logique de soutien à l'innovation, comme le Creative People Lab du constructeur automobile Renault ou I2R (pour "Incubateur d'innovation de rupture ») du département Enerbat de la recherche et développement ( $R$ \& D) d'EDF. Ceux de type "grand public et pro amateurs » se veulent ouverts sur l'environnement local et à des usagers variés, qu'il s'agit d'intéresser aux formes plus personnelles de production, comme la Fabrique d'objets libres à Lyon, qui se revendique être un "laboratoire citoyen de fabrication ».

Quel que soit leur positionnement, ces lieux ont l'ambition commune d'élargir la diffusion des technologies de fabrication numérique, sous une forme de surcroît qui permette de rendre actifs les utilisateurs. Leurs projets, même s'ils peuvent être menés essentiellement par une seule personne, ne sont pas censés être réalisés de manière individualiste. Que ce soit pour créer des drones, des prothèses ou tout objet plus ou moins utile, par exemple un appareil de mesure de la pollution atmosphérique intérieure comme au Nicelab - «laboratoire ouvert» de Nice - ou un "oreiller lumineux» comme au Labfab de Rennes - pour « un réveil tout en douceur grâce à la luminothérapie»-, ces projets sont ainsi le produit de "communautés de pratique» (Wenger, 2005), dans lesquelles les participants se retrouvent dans un engagement mutuel et peuvent apprendre en partageant informations et compétences.

En France, des acteurs gouvernementaux ont marqué un intérêt pour cette dynamique apparemment montante, mais l'ont abordée sous un angle essentiellement économique. L'appel à projets « Aide au développement des ateliers de fabrication numérique », lancé en 2013 par la Direction générale de la compétitivité de l'industrie et des services (DGCIS), est resté aligné sur une vision fortement entrepreneuriale et soucieuse de débouchés économiques ${ }^{(6)}$. Sur les 154 dossiers déposés pour cette aide au financement de projets, 14 ont été sélectionnés, non sans polémique d'ailleurs dans une partie du milieu des fab labs à cause de l'opacité des critères de choix et d'une orientation jugée justement trop entrepreneuriale.

(6) Le site ministériel précisait: « Les projets devront viser le développement économique des entreprises, et à ce titre elles devront proposer des services à destination des entreprises. Dans cette optique, les projets devront s'engager dans un travail de recherche d'une pérennité économique et prévoir de définir et de tester un modèle économique impliquant les entreprises utilisatrices. » 


\section{Au-delà du prisme de l'innovation}

Par la mobilisation conjointe des énergies collaboratives et des ressources technologiques dérivées du numérique, ces lieux expérimentaux peuvent véhiculer une logique d'innovation, mais aussi mettre en acte des réflexions et des aspirations d'un courant d'auteurs qui avaient commencé, à partir des années 60 et 70 , à compléter leur critique du modèle industriel par une esquisse de voies alternatives: Murray Bookchin (1974), sur la possibilité d'une "technologie libératrice »; Ivan Illich (1973), sur la "convivialité» des outils; Fritz Schumacher (1978), sur les "technologies intermédiaires »; André Gorz, sur la défense de la sphère d'autonomie (Gollain, 2009); Ingmar Granstedt (2007; 2010), sur les outils "autonomes".

C'est dans ce type de perspectives et de filiations que fab labs et makerspaces pourraient représenter une forme de projet également politique, avec une tonalité presque émancipatrice. Au-delà de la rhétorique de l'innovation, ils semblent proposer une voie de réappropriation des outils et des activités de production. Ces ateliers visent des performances presque comparables aux équipements industriels, mais en gardant un positionnement proche de l'artisanat, qui peut ainsi être plus adapté aux désirs et aux besoins. Conçus de manière participative, les projets semblent pouvoir devenir plus facilement appropriables par des populations plus ou moins proches.

Sur ce principe, une série de partenaires autour du Fab Lab Network et du Fab Lab Barcelona s'est par exemple lancée dans la conception et la fabrication d'une petite maison (Fab Lab House) capable d'utiliser les ressources de son environnement (le soleil notamment) pour être autosuffisante en énergie. Comme ce projet vise la collectivité la plus large, ses caractéristiques peuvent être récupérées sur un wiki, qui permet de retrouver spécifications et étapes de réalisation ${ }^{(7)}$.

(7) http://wiki.fablabbcn.org/ Fab_Lab_House_Model. Un site lui est également dédié: www.fablabhouse.com/en.

Les organisations qui s'expérimentent semblent suffisamment flexibles pour être capables d'agréger de manière non sélective des contributions variées, tout en portant et en incarnant un modèle collaboratif. Mais l'analogie avec les coopératives ne fonctionne pas complètement, dans la mesure où les participations peuvent être plus fluides et où les projets ne sont pas forcément structurés par des besoins économiques précis. De fait, en France, lorsque le projet vise un public large, le statut associatif est plus souvent privilégié.

Ces expérimentations ont même commencé à s'agréger dans un mouvement qui ambitionne de s'étendre à une échelle mondiale. L'International Fab Lab Association prétend aider à promouvoir, à organiser et à structurer la communauté qui s'est développée ${ }^{(8)}$. Si le nombre d'implantations

(8) « Why we are here», www. fablabinternational.org/fabassociation/why-we-are-here, consulté le 6 avril 2014. augmente notablement, une part de la population peut espérer accéder à de nouvelles capacités de production dans un environnement proche. L'inspirateur des fab labs, Neil Gershenfeld, a lui-même pu appuyer ce type de vision en lui donnant une portée extensive: "Ces laboratoires font partie d'un mouvement maker plus vaste et composé d'adeptes d'un do-it-yourself en version haute technologie, qui sont en train de démocratiser l'accès aux moyens modernes de fabriquer des choses »(Gershenfeld, 2012, p. 48; notre traduction). Il n'est pas seul 
à esquisser cette dynamique et il y a dans ces communautés une forme de conscience que certains ressorts déjà plus ou moins présents peuvent effectivement aider à renverser des dépendances.

\section{Des ressources technologiques aux capacités sociopolitiques}

Derrière la fascination diffuse pour les technologies de fabrication numérique, l'attention et les questions que peuvent susciter les fab labs tiennent aussi aux usages qu'ils semblent permettre. Les enjeux sont certes technologiques, mais surtout ils renvoient aux capacités qui peuvent être construites dans de tels espaces et aux formes de production qu'ils rendent possibles.

\section{Une voie de réappropriation de la technologie}

L'un des arguments qui participent de l'attrait exercé par les fab labs est qu'ils permettraient d'exprimer, voire d'étendre, un potentiel de créativité plus ou moins latent, précisément en élargissant les capacités de fabrication à l'échelle de petits collectifs. Ces communautés cherchent la possibilité de développer leurs propres outils, de concevoir leurs propres objets, en fonction de leurs besoins et de leur imagination.

Par la pratique et une assistance, si nécessaire, ces espaces fonctionnent ainsi en favorisant une forme de réappropriation de certains outils. Dans un tel cadre, le rapport aux technologies peut être envisagé selon une logique plus conviviale, pour reprendre la perspective d'Ivan Illich ${ }^{(9)}$, dans la mesure où elles sont rendues accessibles et maîtrisables, a priori sans discrimination de compétences, et où elles peuvent être plus proches des lieux de vie et des usages. Sur ce schéma, EchoFab à Montréal lie par exemple accès aux technologies et autonomisation: "En transférant les activités de fabrication et de création au cour des communautés, ses membres peuvent acquérir la maîtrise des outils et diffuser les connaissances nécessaires à leur propre autonomisation. L'objectif de démocratisation technologique des fab labs vise aussi à accroître l'accès aux technologies de fabrication pour favoriser la création ${ }^{(10)}$. Avec ces initiatives, peut donc devenir disponible

(9) «L'outil est convivial dans la mesureoù chacun peutl'utiliser, sans difficulté, aussisouvent ou aussi rarement qu'ille désire, à des fins qu'il détermine lui-même. L'usage que chacun en fait n'empiète pas surla libertéd'autruid'enfaire autant» (Illich, 1973, p. 45). (10) www.echofab.org/612-2, consulté le 14 juin 2014. un type d'espaces relativement nouveau où des individus ont la possibilité d'exprimer des désirs créateurs, tout en conservant une appréhension et un contrôle de la finalité des technologies utilisées. Reconstruit, le rapport aux machines apparaît alors plus comme un rapport amateur que professionnel: d'un côté, le niveau d'exigence peut rester élevé, mais, de l'autre, la liberté d'exploration et d'expérimentation est valorisée.

\section{Apprendre en faisant et partager les connaissances}

Outre cette possibilité de favoriser l'expression créative, l'état d'esprit qui imprègne les fab labs met également en avant la stimulation de l'apprentissage. Précisément, c'est par la pratique (la «bidouille» même, pour reprendre le terme qui y circule fréquemment) que l'apprentissage est souhaité, escompté et encouragé dans ce type de lieu. La dimension d'«éducation " est ainsi promue dans une des versions françaises de la charte 
des fab labs: "La formation dans le fab lab s'appuie sur des projets et sur l'apprentissage par les pairs. Vous devez prendre part à la capitalisation des connaissances et à l'instruction des autres utilisateurs »(11). Il s'agit d'apprendre en faisant avec d'autres, qui ont déjà appris dans le fab lab ou ailleurs ( Faire pour apprendre, non pas seul, mais ensemble », comme le revendique le Faclab de l'université de Cergy-Pontoise $\left.{ }^{(12)}\right)$.

(11) http://fablabinternational. org/fr/fab-lab/charte-des-fablabs, consultéle 10 avril 2014. (12)www.faclab.org/apprendreen-faisant, consultéle 12 juin 2014.

Open Bidouille Camp est une initiative qui s'est développée sur ce type de principe, après une première expérimentation le 22 septembre 2012 à Saint-Ouen (Seine-Saint-Denis). L'idée, mêlant la culture open source, l'esprit du do it yourself, la gratuité des échanges, la quête de réparabilité, sous forme d'ateliers thématiques, a été reconduite l'année suivante, en février, au Faclab de Gennevilliers et reprise depuis dans d'autres villes.

Derrière le propos technologique, la logique est donc aussi souvent celle de la capacitation. Certains fab labs portent en effet un discours qui peut rappeler celui de l'éducation populaire. Le projet de Lannion, dans les Côtes-d'Armor, s'est constitué en affichant ce genre d'approche comme l'une de ses orientations constitutives: "L'un des objectifs principaux du fab lab est de donner accès aux citoyens aux connaissances et aux outils nécessaires pour comprendre comment un objet est fabriqué, comment il fonctionne, comment le modifier et comment le réparer. Bref: à se réapproprier des connaissances techniques peu accessibles au grand public ${ }^{(13)}$. Le postulat courant dans ces projets

(13) http://fablab-lannion. org/le-projet/un-lieu-d-edu cation-populaire, consulté le 12 juin 2014. est que les connaissances, même techniques, doivent circuler et qu'elles peuvent être partagées dans l'expérimentation en commun. Le rapport à la technique (de fait, de plus en plus présente dans les environnements les plus quotidiens) et aux biens de consommation tend lui-même à devenir objet de questionnement, de sorte qu'il n'est plus simplement dans la passivité. D’où la valorisation de la dénomination de makers pour les personnes qui s'engagent dans ces activités: ils ne se contentent pas de consommer; ils font. Il en découle également des formes de rapprochement avec l'éducation scientifique et technique en direction des jeunes générations, amenant conjointement des liens avec des réseaux associatifs (locaux ou plus larges) sur ce créneau, comme Les Petits Débrouillards (sous forme de convention au fab lab de Lannion, par exemple).

Dans les activités, il n'y a pas d'assignation de rôle: celui qui a appris est invité à partager ses connaissances avec d'autres et à les accompagner dans leurs propres expérimentations. Ce qui explique le souhait fréquent que ces lieux soient également propices aux rencontres: autrement dit, "[p]rivilégier l'interdisciplinaritéet mettre en contact des personnes d'horizons différents ", pour reprendre les termes avec lesquels sont présentées les valeurs du projet d'Artilect FabLab Toulouse ${ }^{(14)}$.
(14) «Qui sommes-nous? ", www.artilect.fr/420-2, consulté le 12 juin 2014.

\section{Des possibilités nouvelles pour une production entre pairs et proche des territoires}

Du point de vue des réalisations, le modèle est celui d'une production entre pairs où le produit n'est plus envisagé comme une «boîte noire ». Non seulement il s'agit d'en comprendre le fonctionnement, mais aussi de le fabriquer sans être dépendant d'un processus industriel, lourd et donc inaccessible. 
Le rassemblement de machines et de compétences effectué dans ces lieux (re)donne des prises sur les processus de fabrication et (ré)introduit les participants dans des tâches que le système industriel avait opacifiées. Le rapport aux objets devient plus réflexif, notamment parce que les modalités de conception sont (ré)interrogées. Documenter les réalisations et, surtout, laisser ces informations en libre diffusion permet en outre de créer une forme de communs, avec des connaissances ou des expériences qui pourront ensuite être reprises par d'autres.

Vus sous cet angle, les fab labs et makerspaces peuvent devenir une pièce dans la reconquête de prises sur les conditions d'existence, précisément en offrant et en distribuant des capacités de production pour les artefacts constitutifs de la vie quotidienne. Bien sûr, il faut un nombre de participants suffisant et ayant une motivation entretenue pour que ces organisations puissent fonctionner dans la durée.

Certaines initiatives visent d'ailleurs à répandre ces lieux, qui sont aussi des lieux de sociabilité, dans les territoires. Soutenu par les autorités municipales, le projet du Fab Lab Barcelona a été élargi avec le souhait affiché d'installer des fab labs dans différents quartiers et de faire de la ville une «FabCity». A Rennes, dans une inspiration proche, le projet situé au départ à l'Ecole européenne supérieure d'art de Bretagne est devenu celui d'un "labfab étendu » devant permettre d'«infuser en réseau». Avec ces extensions, les fab labs peuvent encore plus se présenter comme un moyen de ramener des outils et des équipements technologiques sur des bases locales, dans un processus propre ensuite à renforcer des capacités pour aider à résoudre des problèmes également locaux, comme l'avait envisagé Neil Gershenfeld: "Et au lieu d'apporter les technologies de l'information aux masses, les fab labs montrent qu'il est possible d'apporter les outils pour le développement de ces technologies, afin de développer et de produire des solutions technologiques locales à des problèmes locaux» (Gershenfeld, 2007, p. 13 ; notre traduction).

\section{Infrastructure productive distribuée et distanciation de l'ordre industriel}

Une autre manière de questionner les potentialités des fab labs est de les replacer dans les transformations des systèmes productifs (Carroué, 2013). A travers ces ateliers de fabrication digitale, ce sont en effet aussi des réseaux sociotechniques qui se réorganisent et dont la portée est à appréhender sous l'angle des chaînes de relations qu'ils peuvent contribuer à reconfigurer (Callon, 1991; Dicken, Kelly, Olds, Yeung, 2001).

\section{Une forme de déconcentration de la production}

Afin de réaliser des économies d'échelle, le modèle industriel a eu tendance à s'organiser autour d'unités productives de grande taille: il permet de faire fonctionner des équipements coûteux dans les mêmes sites, à partir desquels s'effectue ensuite une distribution sur de larges zones géographiques. Fab labs et makerspsaces sortent de cette logique de concentration des moyens de production: les unités sont plus petites, mais la prétention est de pouvoir 
fabriquer des biens équivalents ou presque. Dans des visions comme celle de Neil Gershenfeld, cette fabrication digitalisée et cet équipement productif miniaturisé vont évoluer au point de devenir aussi accessibles et répandues que la micro-informatique.

Avec de telles machines ainsi adaptées, mobiliser de gros investissements paraît moins nécessaire et trouver ou aménager des locaux pour les accueillir peut être moins compliqué. Les imprimantes 3D (en trois dimensions, sur le principe d'ajouts successifs de couches de matériau) incarnent notamment ce type d'espoir : grâce aux avancées dont elles ont bénéficié conjointement à leur environnement numérique, elles changent l'image des processus de fabrication, en ajoutant aussi tendanciellement celle d'une répartition plus large des capacités corrélatives (voir aussi Rumpala, 2013b). Au stade actuel, encore souvent celui de l'expérimentation et de la diffusion progressive, les modèles les plus accessibles servent, certes, plutôt à prototyper et à produire de petits objets très basiques. Des communautés d'acteurs intéressés, pas forcément professionnelles, poursuivent cependant les efforts pour améliorer leur précision et élargir la gamme des matériaux utilisables. Et, de fait, les fab labs jouent souvent un rôle de popularisation et de démonstration, voire de perfectionnement, des capacités de ces machines (rares sont ceux qui n'ont pas d'imprimante 3D ou qui n'ont pas intégré ce type de machine dans leurs projets).

\section{Contournements des structures industrielles}

Fab labs et makerspaces suscitent en outre un intérêt pour les besoins non pris en compte par les industries existantes. Leurs capacités de production (au sens large, puisqu'il peut s'agir aussi de réparation) peuvent être vues comme un moyen de réduire la dépendance à l'égard du système industriel dominant. Par la miniaturisation et la mise en commun des équipements, le coût d'entrée dans la fabrication est abaissé. Les productions et les projets réalisés n'ont pas à chercher des débouchés, puisqu'ils sont motivés par des besoins locaux. Contrairement aux productions destinées à des marchés de masse, celles des fab labs n'ont pas nécessairement besoin d'être standardisées. Neil Gershenfeld envisageait même que dans l'équipement de ces structures, la configuration de base puisse évoluer vers encore plus d'autonomie, avec des machines qui permettent de produire d'autres machines, jusqu'à pouvoir reconstituer l'équipement entier d'un fab lab: "Ce n'est pas une configuration fixe. Le but à terme est de remplacer des éléments du fab lab par des éléments fabriqués dans le fab lab, jusqu'à ce qu'au bout du compte les fab labs se reproduisent eux-mêmes" (Gershenfeld, 2007, p. 12; notre traduction).

Si ce type de logique est prolongé, au moins pour certaines productions, d'anciens circuits économiques peuvent donc se trouver contournés. Ces lieux, face aux structures industrielles, engagent à la fois dans une désintermédiation et une réintermédiation: ils se positionnent en fait comme de nouveaux intermédiaires, opérant à l'écart de logiques strictement professionnelles.

Dans certains fab labs, la conscience des ressources limitées de la planète amène également à essayer de contourner l'enjeu des difficultés d'accès 
aux matériaux en promouvant la récupération. Avec une telle logique, ces initiatives se rapprochent alors d'une économie " circulaire " ou d'une économie " de fonctionnalité », où l'une des préoccupations est de pouvoir boucler les cycles d'utilisation des matières ${ }^{(15)}$. En Belgique, RElab, un fab lab monté à Liège, affiche ainsi comme spécificité "l'utilisation de matériaux de récupération comme matière première et [...] l'étude de nouveaux procédés sociaux, créatifs et économiques $d$ 'upcycling [une façon de valoriser des déchets], en liaison avec les nouveaux moyens de fabrication et de communication numérique » ${ }^{(16)}$. Dans son portefolio de projets, le Labfab de Rennes en propose un (Plabs recycle, en référence à l'acrylonitrile butadiène styrène, un polymère thermoplastique) qui "prend les paris de transformer à moindre coût et à domicile les chutes en filament",

(15) Nicolas Buclet (2014) met ainsi les fab labs dans le sixième et dernier type de sa typologie d'économie de fonctionnalité.

(16) http://relab.be, consulté le 12 juin 2014.

(17) http://www.labfab.fr/ portfolio/plabs-recycle, consulté le 12 juin 2014. utilisable donc pour de l'impression $3 \mathrm{D}^{(17)}$.

La rationalité économique, enfin, est différente, dans la mesure où les $f a b$ labs ne visent pas forcément des objectifs marchands: ce qui est produit n'est pas nécessairement destiné à être mis en vente sur un marché et acheté par des « consommateurs ». En s'écartant des préoccupations de propriété, voire en les remettant en cause, ces activités prolongent des affinités avec une "culture du don technologique " (Oliveri, 2011) qui a sous-tendu le développement des logiciels libres. Dans un rapport proche, la dynamique favorable à l'ouverture qui s'était développée dans le domaine des logiciels, sous la bannière de l'open source, tend à s'élargir aux matériels, avec non seulement des initiatives qui poussent en ce sens, mais aussi des projets qui visent à des réalisations concrètes (en électronique, la carte Arduino est par exemple l'une des plus connues pour ses multiples applications possibles, utilitaires ou purement ludiques, intéressant donc de nombreux fab labs).

\section{Déplacements dans l'appréhension du « travail »}

Fab labs et makerspaces sont des lieux a priori non hiérarchisés, où s'agrègent des contributions volontaires. A la confluence du do it yourself et des échanges entre pairs, également dans une forme de prolongement de dispositions d'esprit communes dans le milieu hacker (Himanen, 2001), le fonctionnement des fab labs et des makerspaces laisse entrevoir une manière relativement originale de concevoir le travail (au moins par rapport à sa réification tendancielle et à sa réduction à un emploi fournissant une rémunération).

S’il y a du travail dans ces lieux, il n'y est pas envisagé de manière abstraite et parcellisée. Quand il s'agit de fabriquer un objet, celui-ci est le plus souvent envisagé de sa conception jusqu'à sa réalisation. Par contraste avec des formes industrielles qui ont pu être critiquées pour leurs tendances aliénantes, le travail réalisé (re)gagne du sens, dans la mesure où les membres des fab labs peuvent ainsi participer à l'ensemble d'un processus, y faire valoir leurs attentes et y insérer leurs idées. Pour ces participants, l'investissement personnel paraît pouvoir avoir un débouché tangible, à une échéance qui peut, certes, être plus ou moins longue. Les projets ne sont pas contraints par un temps imparti et chacun peut espérer avoir la possibilité de suivre le rythme qu'il souhaite. 


\section{Conclusion : un projet déjà en voie de normalisation?}

Si le modèle des fab labs et des makerspaces se répand, c'est qu'il séduit (en attirant aussi l'attention des médias, d'ailleurs), mais, dans la phase actuelle, ce sont toujours des espaces interstitiels, dans lesquels un nombre encore réduit d'acteurs est engagé dans des interactions plus ou moins régulières et plus ou moins organisées.

S'il apparaît porteur de potentialités sociopolitiques, et pas seulement techniques, le développement de ces structures est toutefois aussi chargé en ambivalences. De tels lieux peuvent être effectivement des intermédiaires facilitant l'accès à la fabrication, mais quand les grands acteurs industriels et publics manifestent un intérêt à leur égard, c'est souvent avec des schémas intellectuels qui les réinscrivent dans les mêmes logiques de développement économique que celles qui ont marqué la fin du $\mathrm{Xx}^{\mathrm{e}}$ siècle (typiquement à la manière de l'appel à projets engagé sous l'égide du ministère du Redressement productif). Les expérimentations sont donc aussi exposées à des formes de normalisation.

Autre question importante: savoir qui finance et comment, car certaines modalités de financement (subventions, partenariats industriels-privés, facturation de services, etc.) peuvent notablement influencer les orientations adoptées et introduire des contraintes, contribuant ainsi à détourner des valeurs originelles. Un tel déplacement peut paraître d'autant plus aisé que, dans ces initiatives, le travail tend à être organisé sur le mode du " projet ». Des motivations qui pouvaient paraître émancipatrices peuvent en définitive se trouver elles aussi canalisées ou absorbées dans la "cité par projet », dont Luc Boltanski et Eve Chiapello (1999) avaient repéré le rôle dans l'installation d'un "nouvel esprit du capitalisme», ce qui peut augurer d'une facilité à s’insérer dans un système capitaliste en évolution et en recherche perpétuelle de voies d'adaptation. De fait, le modèle des fab labs permet davantage de flexibilité dans la production et peut être récupéré et instrumentalisé par de grandes organisations industrielles, qui peuvent les exploiter comme des réservoirs (externalisés) non seulement de flexibilité, mais aussi de créativité et d'idées.

Qu'est-ce qui compte alors? Peut-être pas tellement leur nombre (encore relativement faible), mais l'imaginaire qui est réouvert. Ces espaces portent potentiellement une évolution du rapport aux objets, aux machines, aux savoirs professionnels. Ils contribuent à installer une autre vision des cycles productifs. La production d'artefacts ne paraît plus réservée aux usines ou à l'univers industriel et les participants deviennent coproducteurs ou "prosommateurs » (à la fois producteurs et consommateurs; Beaudouin, 2011; Ritzer, Dean, Jurgenson, 2012).

Cet accès à une fabrication plus locale est en outre porteur d'un changement du rapport au temps et à l'espace. Sur les deux derniers siècles, la logique industrielle a en effet aussi contribué à organiser les rythmes de vie et de travail, mais également les territoires. Cette logique était devenue une "dimension de la mondialisation», comme le rappelait le sociologue Anthony Giddens: "L'industrie moderne est intrinsèquement basée sur des divisions du travail, non seulement au niveau des tâches, mais aussi de 
la spécialisation régionale en fonction du type d'industrie, de savoir-faire et de la production des matières premières » (Giddens, 1994, p. 82).

Avec l'émergence d'un nouveau type de système productif, la domination de l'ordre industriel paraît plus incertaine. Et s'il est possible de parler de "système technicien" (Ellul, 2004), ce dernier semble perméable à d'autres logiques que des logiques industrielles, voire laisse entrevoir une nouvelle trajectoire possible (à moins que ce ne soit un nouveau "front pionnier " d'un capitalisme toujours adaptatif, facilitant le développement d'un agile manufacturing; Mourtzis, Doukas, 2014).

Ce qui est également intéressant, ce n'est pas seulement ce qui est fait dans ces lieux (les productions), mais comment (les processus). Les participants mettent en œuvre une éthique (qui n'est pas sans rappeler l'éthique hacker, comme on l'a vu) articulant une série de valeurs et favorisant de fait l'échange et le partage. Dans l'esprit des makerspaces, l'enjeu n'est plus de posséder des moyens de production, mais d'y accéder.

Mais pour y faire quoi? De nouveaux gadgets? Le résultat dépendra de la dose de réflexivité qui sera appliquée à des pratiques multiples encore émergentes. D'autant qu'en effet les technologies numériques ont aussi leur lot de contraintes et qu'en arrière-plan continuera à peser la problématique écologique (Flipo, Deltour, Dobré, Michot, 2012) et son incontournable lot de dépendances, spécialement par rapport aux approvisionnements en ressources matérielles. 


\section{BibLIOgRAPHIE}

Anderson C., 2012, Makers: The new industrial revolution, New York, Crown Business.

Beaudouin V., 2011, « Prosumer », Communications, n 88, p. 131-139.

Boltanski L., Chiapello E., 1999, Le nouvel esprit du capitalisme, Paris, Gallimard.

Bookchin M., 1974, Vers une technologie libératrice, Paris, Librairie Parallèles.

Buclet N., 2014, «L'économie de fonctionnalité entre éco-conception et territoire: une typologie », Développement durable et territoires (en ligne), vol. $5, \mathrm{n}^{\circ} 1$. Site: http://developpement durable.revues.org/10134.

CallonM.,1991, « Réseauxtechnico-économiques et irréversibilités », in Les figures de l'irréversibilité en économie, Robert Boyer, Bernard Chavance, Olivier Godard (dir.), Paris, Editions de l'EHESS.

Carroué L., 2013, La France: les mutations des systèmes productifs, Paris, Armand Colin.

Dicken P., Kelly P. F., Olds K., Yeung H. W., 2001, "Chains and networks, territories and scales: Towards a relational framework for analysing the global economy ", Global Networks, vol. $1, \mathrm{n}^{\circ} 2$, p. 89-112.

Ellul J., 2004, Le système technicien, Paris, Le Cherche Midi.

Eychenne F., 2012, Fab lab : L'avant-garde de la nouvelle révolution industrielle, Limoges, FYP Editions.

Flipo F., Deltour F., Dobré M., Michot M., 2012, Peut-on croire aux TICvertes? Technologies numériques et crise environnementale, Paris, Presses des Mines.

Gershenfeld N., 2007, Fab: The coming revolution on your desktop from personal computers to personal fabrication, New York, Basic Books.

Gershenfeld N., 2012, " How to make almost anything: The digital fabrication revolution ", Foreign Affairs, vol. 91, n 6, p. 43-57.

Giddens A., 1994, Les conséquences de la modernité, Paris, L’Harmattan.
Gollain F., 2009, « Penser la question des outils avec André Gorz », EcoRev', n³3, automne.

Gorz A., 2008, Ecologica, Paris, Galilée.

Gorz A., 2007, « Penser l'exode de la société du travail et de la marchandise ", Mouvements, $\mathrm{n}^{\circ} 50$, p. 95-106.

Granstedt I., 2007, Du chômage à l'autonomie conviviale, Lyon, A plus d'un titre.

Granstedt I., 2010, L'impasse industrielle: un mondeà réoutiller autrement en tous lieux, Lyon, A plus d'un titre.

Himanen P., 2001, L'éthique hacker et l'esprit de l'ère de l'information, Paris, Exils.

Illich I., 1973, La convivialité, Paris, Le Seuil.

Mourtzis D., Doukas M., 2014, « The evolution of manufacturing systems: From craftsmanship to the era of customisation ", in Vladimir Modrák, Pavol Semančo (eds.), Handbook of research on design and management of lean production systems, Hershey, IGI Global, p. 1-29.

Oliveri N., 2011, « Logiciel libre et open source: une culture du don technologique ", Quaderni, $\mathrm{n}^{\circ} 76, \mathrm{p} .111-119$.

Ritzer G., Dean P., Jurgenson N., 2012, « The coming of age of the prosumer ", American behavioral scientist, vol. 56, $\mathrm{n}^{\circ}$ 4, p. 379-398.

Rumpala Y., 2013a, "Formes alternatives de production énergétique et reconfigurations politiques: la sociologie des énergies alternatives comme étude des potentialités de réorganisation du collectif ", Flux, n ${ }^{\circ} 92$, p. 47-61.

Rumpala Y., 2013b, "L'impression tridimensionnelle comme vecteur de reconfiguration politique », Cités, n 55, p. 139-162.

Schumacher E. F., 1978, Small is beautiful: une société à la mesure de l'homme, Paris, Contretemps-Le Seuil.

Wenger E., 2005, La théorie des communautés de pratique: apprentissage, sens et identité, Laval, Presses de l'université Laval. 